\title{
The Rigid-flexible Coupling Dynamics Analysis of the Powder Filling Quantity Control Mechanism Based on the ADAMS
}

\author{
Guihe Wang ${ }^{1}$, Liu Ying ${ }^{1}$, Na Cui ${ }^{2}$, Yiqi Wang ${ }^{3, *}$ and Yijing Zhang ${ }^{1}$ \\ ${ }^{1}$ Mechanical Engineering \& Automation Department, Eastern Liaoning University, Dandong, China \\ ${ }^{2}$ Faculty of Engineering Department, Tieling Normal College, Tieling, China \\ ${ }^{3}$ International Department, Shenyang University, Shenyang, China \\ Corresponding Email: yiqiwang001@163.com
}

\begin{abstract}
Powder filling quantity control mechanism is an important part of medicine machine. The geometric model of the filling quantity control mechanism was established by Pro/E based on the NJP1200 capsule filling machine. By Lagrange multiplier method, the equation of movement characteristics was established. And also, the rigid-flexible coupling model of the mechanism was established by ADAMS, Based which, the simulation of displacement, velocity and acceleration curve were obtained. In the end, the change rule of CAM contact force was analyzed. It showed that ADAMS is an effective method in simulation the dynamic characteristics of mechanism.
\end{abstract}

\section{Introduction}

Automatic capsule filling machine is a key capsule preparation filling equipment. There are more than 7500 pharmaceutical enterprises in our country and $99 \%$ of the capsule filling machine belongs to the plunger type filling machine. Its advantage is easy to operate, safe and good sealing, but the powder filling quantity accuracy is greatly affected by the mechanical structure, powder liquidity and filling environment. At present, the drug companies are mostly using NJP1200 type, NJP2000 type and NJP3200 type capsule filling machine in our country. More advanced foreign capsule filling machine are represented G120N of Italy MG2, ZANAS125/40F of IMA company and GKF2000 of Germany BOSCH [1].

Capsule filling machine moves complex, including continuous movement, intermittent movement, linear movement, rotational movement. So the movement precision is high. In recent years, the related technology of the capsule filling machine design and manufacture has been mastered through the introduction, digestion and absorption from abroad in our country, but the powder filling quantity control technology remains to be further research and development. Dynamics research is doing based on ADAMS of the powder filling quantity adjusting mechanism to optimize the the structure and parameter of the related parts of filling quantity control, improving the design level of the fully automatic capsule filling machine in our country $[2,3]$.

\section{The working principle of capsule filling machine}

Capsule filling machine is mainly composed of transmission mechanism, capsule delivery mechanism, capsule separation mechanism, lock capsule mechanism, module rotary and powder filling quantity control mechanism. Transmission mechanism completed the corresponding movement by gear motor through the chain corresponding CAM mechanism, as shown in Figure 1. 


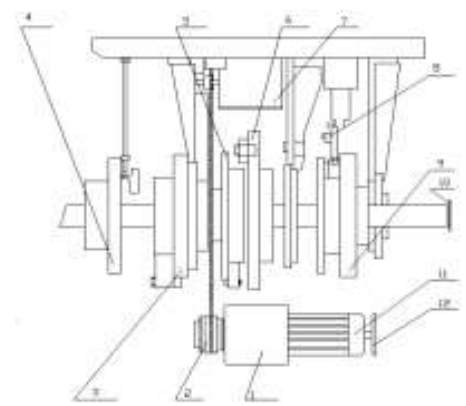

1-Reducer, 2- Safety clutch, 3,4,5,6,8,9- CAM, 7- Dividing box, 10- Dial, 11- Motor, 12- Motor handwheel

Fig. 1. Driving sketch of capsule filling machine.

The main shaft in transmission mechanism is installed six CAM mechanisms by holding linkage: CAM 3 completes lock capsule function; CAM 4 completes eliminating waste capsule function; CAM 5 completes combining capsule function; CAM 6 completes powder filling quantity control and filling function; CAM 8 completes separating capsule function and CAM 9 completes delivery capsule function. Intermittent mechanism includes ten locations and six location controlling the movement of the module and the powder filling quantity mechanism respectively. Through intermittent mechanism the module rotary movement and filling movement coordinate in order to complete capsule delivery, capsule separation, capsule filling, capsule elimination, capsule combination, capsule output and other functions in different location orderly to ensure that each location runs accurately and smoothly [4].

The powder filling movement is an important part of the movement of the capsule filling machine and can affect the powder filling quantity accuracy. Powder filling quantity control is completed by the filling CAM, linkage and so on, its working principle is shown in Figure 2.

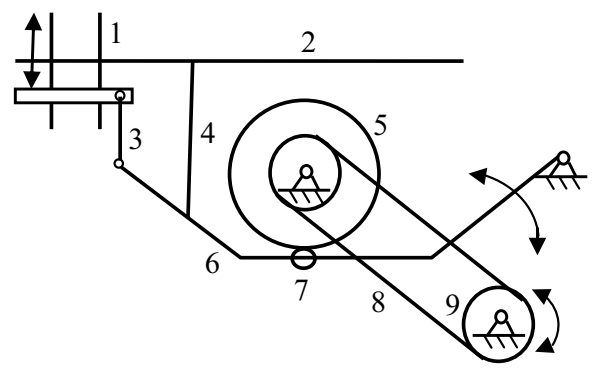

1-Push rod, 2Big board, 3-Buide rod, 4-Spring, 5-Filling CAM, 6-Connecting rod, 7-Roller, 8-Roller chain, 9-Sprocket

Fig. 2. Driving sketch of powder filling structure.

Gear motor drives the sprocket (9) and the rotation will be passed to the filling CAM (5) by the chain (8). The swinging movement of the connecting rod (6) is completed by the roller (7) on the connecting rod with the rotation of the filling CAM. Guide rod (3) and connecting rod (6) are linked together through joint bearing to make the guide rod (1) completing linear movement, namely filling movement.

\section{The rigid-flexible coupling model of the filling quantity control mechanism}

\subsection{The motion equations}

The dynamics model of powder filling quantity control mechanism was set up based on the theory of Lagrange equation [5], namely

$$
\begin{aligned}
& \mathrm{q}_{\mathrm{i}}=[\mathrm{x}, \mathrm{y}, \mathrm{z}, \psi, \theta, \varphi]_{\mathrm{i}}^{\mathrm{T}} \\
& \mathrm{q}=\left[\mathrm{q}_{1}^{\mathrm{T}}, \cdots, \mathrm{q}_{\mathrm{n}}^{\mathrm{T}}\right]^{\mathrm{T}}
\end{aligned}
$$

The movement equation of the powder filling quantity mechanism was gotten combined with the generalized coordinates expression by Lagrange multiplier method, namely: 


$$
\frac{\mathrm{d}}{\mathrm{dt}}=\left[\frac{\partial \mathrm{T}}{\partial \dot{\mathrm{q}}}\right]^{\mathrm{T}}-\left[\frac{\partial \mathrm{T}}{\partial \mathrm{q}}\right]^{\mathrm{T}}+\mathrm{f}_{\mathrm{q}}^{\mathrm{T}}+\mathrm{g}_{\dot{\mathrm{q}}}^{\mathrm{T}}=\mathrm{Q}
$$

When the filling quantity mechanism is fully restrained: $f(q, t)=0$; When the filling quantity mechanism is unfully restrained: $g(q, \dot{q}, t)=0$. Among them:

$\mathrm{T}$ - kinetic energy of the filling quantity mechanism;

Q- generalized coordinates array of the filling quantity mechanism;

q- the Laplace multiplier array corresponding with the fully restrained;

$\mathrm{M}$ - the Laplace multiplier array corresponding with the unfully restrained;

$\dot{\mathrm{q}}$

generalized velocity array of the filling quantity mechanism.

\subsection{The ADAMS model of the powder filling quantity control mechanism}

Powder filling quantity control mechanism is composed of dose plate, filling rod, coppering, etc. Powder is compacted layer-by-layer and filled into the capsule body by 6 groups filling bar according to the demand of powder filling quantity. Spindle drives filling CAM rotation and CAM drives column through the linkage doing up and down reciprocating motion to realize the powder filling. The depth of the filling rod inset the dose plate can be adjusted by the knob to adjust to the volume of powder filling. Filling mechanism model was established based on Pro/E and imported into the ADAMS, as shown in Figure 3.

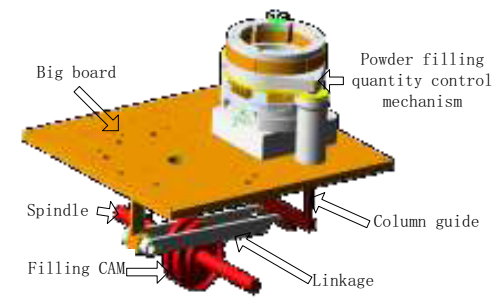

Fig. 3. Model of filling machine of ADAMS.

In order to reduce the constraints imposed in ADAMS, when rigid body is set in MECH /pro, the synchronous movement parts are set to a rigid body. Big board is set to be fixed joint; Connecting rod and big board are set to be revolute joint; Roller and CAM are imposed collision constraints, Roller and connecting rod are connected through the revolute joint.

\subsection{The rigid-flexible coupling model}

Because there is no such thing as pure rigid body, the commonly used method is to simplify the flexible body for the rigid body in Mechanism Simulation for convenience. But the gap of the simulation results and the actual results is larger [6-8]. Because of the flexible body simulation computation time is long, therefore, in the simulation only the important components in the system will be treated as flexibility and other parts as the rigid body, namely the rigidflexible coupled simulation analysis, the process as shown in Figure 4. 


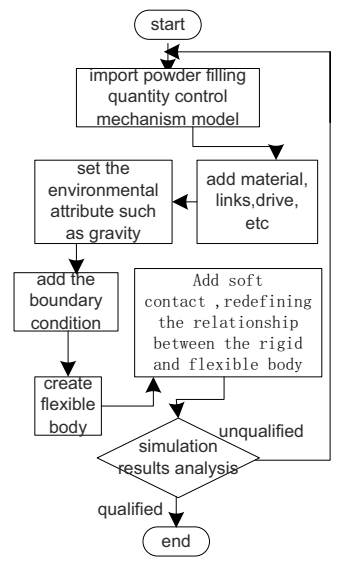

Fig. 4. Analysis chart of rigid-flexible coupling model in ADAMS.

The necessary simplification was done in the process of establishing the rigid-flexible coupling model of the powder filling quantity control mechanism in order to reduce the simulation computation. Two $30 \mathrm{~N}$ forces were added at the top of the CAM rod to instead of the synthesis reaction of column guide on the CAM and corresponding environment variables, material properties, links and drive, etc. were set up. The rigid filling CAM was translated into flexible body using Adams/flex module and according to the actual working conditions of the CAM, the rigid-flexible coupling body was set up as shown in Figure 5.

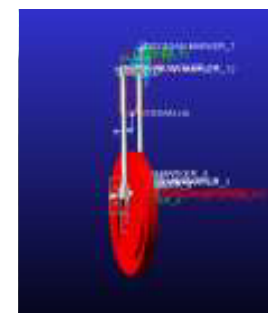

Fig. 5. Structuring of rigid-flexible coupling model.

\section{The analysis of simulation results}

Based on the established rigid-flexible coupling model and using the Adams Simulation function, kinematics and dynamics simulation was done by Adams/Postprocessor module. The movement simulation was done by setting the

CAM speed at $90^{\circ} \mathrm{/s}$, end time of the simulation at $5 \mathrm{~s}$ and steps to 100 . The displacement, velocity and acceleration curve along with the time change for the powder filling quantity control mechanism vertical movement are shown in Figure 6 (a), (b), (c) f change over time.

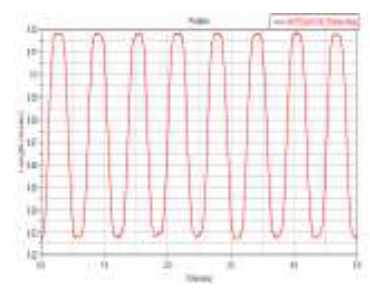

(a) displacement curve

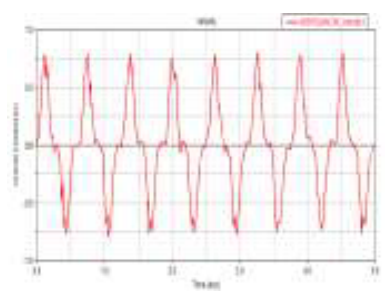


(b) speed curve

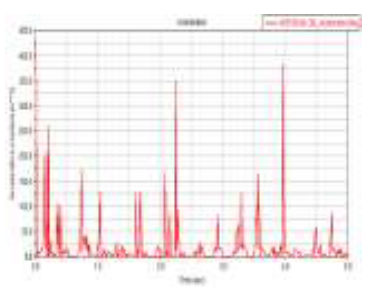

(c) acceleration curve

Fig. 6. Curve of follower.

From the filling quantity mechanism motion curve shown in Figure 6 (a), (b), (c), Comparison data between simulation and design data is shown in table 1 .

Table1. Comparison data between simulation and design data.

\begin{tabular}{|l|l|l|}
\hline project & $\begin{array}{l}\text { simulation } \\
\text { data }\end{array}$ & $\begin{array}{l}\text { design } \\
\text { data }\end{array}$ \\
\hline displacement $(\mathrm{m})$ & 0.09 & 0.09 \\
\hline speed $(\mathrm{m} / \mathrm{s})$ & $-0.7 \sim 0.79$ & $-0.8 \sim 0.8$ \\
\hline acceleration $\left(\mathrm{m} / \mathrm{s}^{2}\right)$ & $0 \sim 50$ & $0 \sim 50$ \\
\hline
\end{tabular}

The simulation displacement is $0.09 \mathrm{~m}$ in agreement with the theoretical design data from table 1; Simulation speed is in the range of $0.7 \sim 0.79 \mathrm{~m} / \mathrm{s}$ basic consistent with theoretical design parameters. Curve has pause phenomenon in zero and In this position the load regulation mechanism motion speed close to zero consistent with intermittent mechanism control; Simulation acceleration value is $0 \sim 50 \mathrm{~m} / \mathrm{s} 2$ consistent with the theoretical design value, but the simulation curve has fluctuations related to the unsmooth CAM contour line surface. ADAMS simulation kinematics performance curve of powder filling quantity control mechanism and practical application motion characteristics of powder filling quantity control mechanism are basic identical with each other.

The surface contact force curve of filling CAM changing with time is shpwn in Figure 7 (a), (b), (c).

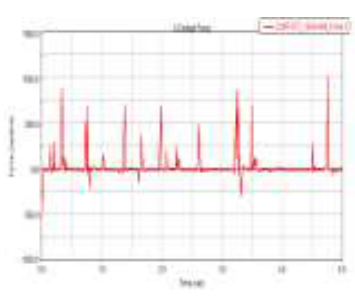

(a) $\mathrm{X}$ direction force curve

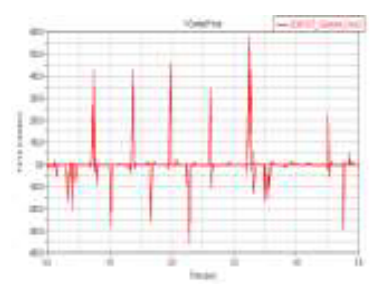

(b) Y direction force curve

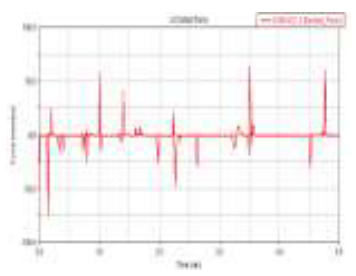

(c) $\mathrm{Z}$ direction force curve

Fig.7. Contact force curve of cam surface. 
The maximum contact force in the $\mathrm{X}$ direction is $1006.8 \mathrm{~N}$ appeared in $4.7 \mathrm{~s}$ moment and minimum value is $501.07 \mathrm{~N}$ appeared in the 0 s moment from Figure 7 (a), (b), (c). The curve is instantaneous fluctuation and the average force is about $0 \mathrm{~N}$; The maximum contact force in the $\mathrm{Y}$ direction is $589.31 \mathrm{~N}$ appeared in $3.5 \mathrm{~s}$ moment and minimum value is $354.61 \mathrm{~N}$ appeared in the $2.3 \mathrm{~s}$ moment. The curve is instantaneous fluctuation and the average force is about $0 \mathrm{~N}$; The maximum contact force in the $\mathrm{Z}$ direction is $65.68 \mathrm{~N}$ appeared in $3.5 \mathrm{~s}$ moment and minimum value is $-749.65 \mathrm{~N}$ appeared in the $0.1 \mathrm{~s}$ moment . The curve is instantaneous fluctuation and the average force is about $0 \mathrm{~N}$; It is can be seen from the graph that although the contact force change is very frequent in simulation environment, the maximum forces value are all in a very small time scale and they are in line with the requirements of the CAM contact force by finding some information.

\section{Summary}

Capsule filling machine is a typical high speed, light load and complex movement equipment. It is a difficult point to research on powder filling quantity control. The rigid-flexible coupling model of the powder filling quantity control mechanism for NJP1200 type capsule filling machine was established based on Pro/E, ADAMS and the simulation analysis was also carried on. (1) The displacement, velocity and acceleration curve were obtained compared with the actual parameters. As a result, the simulation curve corresponds with the actual mechanism motion characteristics. (2) The contact force change regular was obtained based on the CAM mechanism contact force curves of the different direction. It is shown that the rigid-flexible coupling model based on ADAMS is an effective method of mechanism dynamic properties analysis.

\section{Acknowledgement}

This paper is supported by Excellent Talents Supporting Plan in Liaoning province(the first level, LR2013062); Public Welfare Projects in Liaoning province(2015004017); Industrial Research Project of Dandong city Technology Bureau (14207) and Eastern Liaoning University Scientific Research Fund Project(2015GG006).

\section{References}

1. H.Zh .Tang, Zh.Yao, Comparison Between Domestic and imported capsule filling machine,Mechanical and Electrical Information.1,137(2010).

2. G.H.Wang, Y.G. Zhang,T. B.Yu and W.Sh. Wang, Dynamics Simulation of Capsule Filling Machine Based Virtual Prototype, Applied Mechanics and Materials. 127(2012).

3. G.H. Wang, F.Xiao and W.Sh.Wang, Research on Capsule Filling Machine Based on Virtual Prototype, Advanced Materials Research. 411(2012).

4. G.H.Wang, Dynamic Simulation Rresearch of the Fully Automatic Capsule Filling Machine Based on ADAMS, Mechanical Design and Manufacturing. 6(2012).

5. W.H. Kim,T.W. Park, Study of optimization of the barrel cam in a paper-cup-forming machine, Journal of Mechanical Science and Technology.(2012).

6. Zh.Y. Wan, P.X. Li, X.H. Zhuang. Elastic Dynamics Analysis of CAM Mechanism Based on ADAMS, Journal of Dalian Jiaotong University. 1(2010).

7. D.Zhang, J.Zhang, W. Zhang, Research on Cylindrical CAM modeling method Based on Pro/E Relation, Mechanical Design and Manufacturing. 5(2012).

8. Q. Tang, CAM Mechanism Analysis and Dynamic Simulation Based on ADAMS, Journal of Anhui University of Science and Technology (natural science edition).4(2010). 